\title{
Semi-Automated Shoreline Extraction in Satellite Imagery and Usage of Fractals as Performance Evaluator
}

\author{
Sinan O. Altinuc, Ali S. Keceli, and Ebru A. Sezer
}

\begin{abstract}
Analysis of shoreline detection has importance in many investigations undertaken by coastal zone and coastal management studies. These studies require tracking changes in shorelines to reach many objectives such as detecting erosion and land mass movements, discriminating land and sea and etc. At the same time shorelines are important features to display dynamic nature of Earth's surface.

In this paper a novel shoreline extraction method and use of fractals as a performance evaluator are proposed. As a first step of shore line extraction, blurring is done on shoreline image to reduce noise. Then variance map calculation and thresholding are applied. In second stage, a series of morphological binary image processing techniques are performed. After user feedback, boundary of the resulting connected component is extracted. Performance evaluation of the proposed method is done by using fractal values. Evaluation is done by matching calculating fractal values of extracted lines and fractal values of handrawn shorelines. A high correlation has been seen between fractal values of computed and handrawn shorelines.Considering the ability of fractal geometry to express natural entities, fractal dimension is contributed as a performance metric.
\end{abstract}

Index Terms - Shoreline extraction, fractal, performance evaluation, image processing.

\section{INTRODUCTION}

Shoreline can be defined as the fringe of land at the edge of sea or river. Shoreline detection studies have many important uses and applications in geological sciences such as discrimination of land and sea, detecting erosion and obtaining information about the characteristics of land masses. Land sea segmentation is previously performed manually by cartographers. With the advancement in satellite and aerial imaging technologies, shoreline detection between land and the sea can be obtained automatically. As image processing and remote sensing methods emerged, some methods were developed for automatic shoreline detection. ${ }^{1}$

Some previous researches [1]-[3] based on the color or gray level difference between land and sea use thresholding and adaptive thresholding methods to categorize land and sea. However, these methods work under the assumption the sea regions are darker which may not be true in several cases.

Di and Li [4] proposed region-based approach. Di and Li. [4] presents semi-automatic method that takes feedback from user to extract shoreline flawlessly.

Another group of researches in this area are based on

Manuscript received September 1, 2013; revised November 5, 2013

The authors are with the Computer Engineering Department of Hacettepe University, Ankara, Turkey (e-mail: sinanonur @ hacettepe.edu.tr, aliseydi.keceli@hacettepe. edu.tr, ebru@hacettepe.edu.tr). texture properties of land and sea. These studies uses supervised machine learning to classify land and sea by using remote sensing image features [5]. There are statistical models to perform segmentation [6]. Li and $\mathrm{Yu}$ [6] use local boundary optimization to extract shoreline. This method uses OTSU [7] segmentation to perform a rough estimation of the statistical properties of the land and sea and a better segmentation is performed by using this assumption and estimation. Lui and Jezek [8] proposed another statistical method that uses locally adaptive thresholding and Canny edge detection. General purpose of edge detection is to reduce the amount of data in an image keeping its structural properties. Canny Edge detection is an edge detection method that performs well optimizing detection localization and number of responses criteria [9].

In this study we propose a method that utilizes the statistical properties of the image. Local variance of the input images are calculated based on saturation values with a sliding window approach, then a variance map is created. After normalization on variance map, OTSU adaptive thresholding is performed. To clean noise and irregularities in the variance map such as waves and ships in the image, a set of binary image processing operations are performed. Human interaction is needed to mark a single point in the sea region. Finally boundary detection is used to complete shore detection.

\section{PROPOSED METHOD}

Our method includes several stages, these stages are preprocessing, color space conversion, saturation channel selection, variance map calculation, thresholding, post processing user correction and boundary detection. Stages are given in Fig. 1 with effectuation order.

\section{A. Preprocessing}

In preprocessing step, Gaussian filter is used to decrease the effect of the noise and prevent the high local variance values caused by single or small group of pixels. Throughout the examination on the land-sea images it is observed that saturation value in HSV [10] color space provides better discrimination of land and the sea rather than regular grayscale values. So image color space is converted to HSV from RGB and ignoring the Hue and Value channels, only the saturation channel, which is the depth and purity of color, is taken as grayscale image. Then the Image is reversed so that the land regions correspond to the higher values.

Sea regions tend to have a low value difference between pixels whereas land regions tend to have a higher value difference. This is usually caused by roughness and variety of material on land. This property of land results in a higher 
variance for sections taken from land regions than sections taken from sea regions. Sections that contain both land and sea parts have even higher local variances due to value differences between land and sea. This variance difference property is used to discriminate land and sea regions.

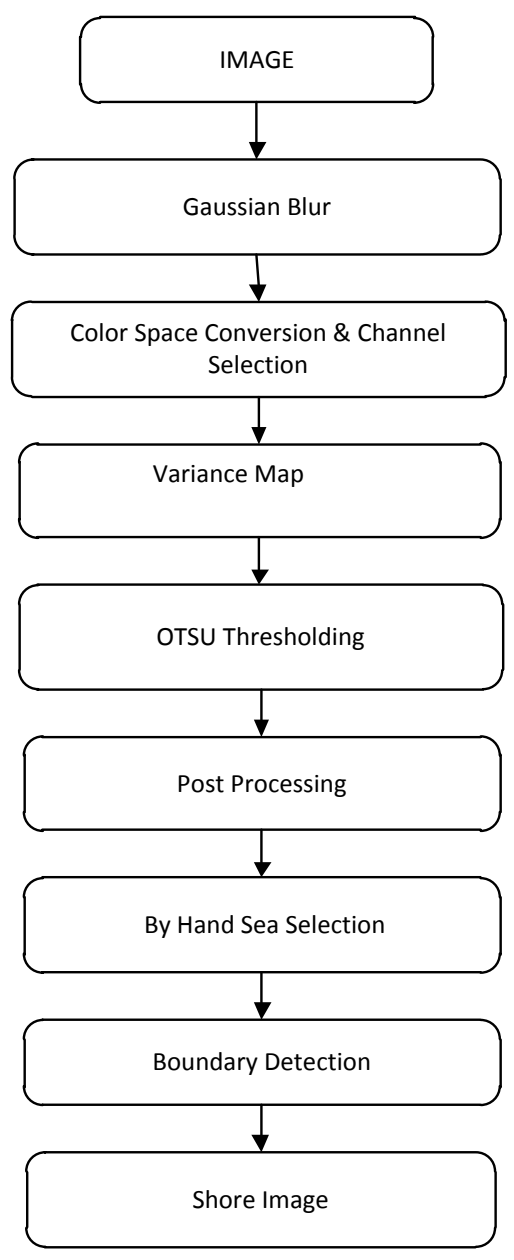

Fig. 1. Steps of Proposed method.

\section{B. Variance Map}

After preprocessing step, variance map of the image is calculated. Variance map is a reconstruction of an image according to their local variance values. Local variance of a point can be found by calculating the variance of pixels in a frame centering the point. Definition of the variance is given in Eq. (1).

$$
\operatorname{var}(X)=\frac{\sum_{i=1}^{N}\left(X_{i}-\mu\right)^{2}}{N-1}
$$

where $\mu$ is the mean on the values,.$N$ is the number of the pixels in a frame (window size). We can generate a variance map for the image using a moving a $n \times n$ frame over the image where $\mathrm{n}$ is an odd number $N$ is calculated as: $N=n \times n$. In this case a $5 \times 5$ frame is used $(n=5)$. The calculated variance value is taken as the value for pixel in the center of the frame.

The calculated variance value corresponds to the pixel value for the variance map image. After constructing the whole variance map the values are normalized. After normalization, highest valued pixel corresponds to the highest possible value in the image. Even after this normalization the variance map is barely visible. The image is further enhanced by multiplying the whole image by a coefficient which can be taken as parameter. For this experiment 20 is used as the parameter.

\section{Thresholding and Binarisation}

After the variance map is created correctly to discriminate the borders of the land and sea mass, global thresholding is enforced with OTSU method. Otsu method [4] is a clustering based thresholding method. This method based on finding the best threshold that separates pixels into 2 classes. Threshold is found by calculating the probabilities of intensity levels and finding the threshold which minimizes intra class variance and maximizes inter-class variance.

After thresholding the image is converted to a binary image. The thresholding and binarisation process provides a rough estimation of land and sea regions. To improve accuracy some binary image processing operations are applied. Purposes of these operations are to basically remove small objects that have no connection to the shoreline, repair possible broken links in the shoreline and thus reduce the error. Morphological dilation, bridging, majority and thinning binary image operations are performed with the given order [10], [11]. After this point to remove unwanted small objects, pixels that have fewer than 4 neighbors are removed from binary image with binary opening. Since our purpose is detection of shoreline that separates land and sea masses, the water masses covered by the land such as lakes are discarded and are marked as a part of land. In this case we can assume that any mass fully covered by land mass should be a part of the land mass itself. The holes in the binary images are filled with 1 's under this assumption.

\section{Manual Land-Sea Selection}

Except some special cases we have a one large connected component of 1's in final binary image. This connected component represents land and zeros represent sea regions. However some 0 regions can be seen where they supposed to be marked as land region. This is caused by the land regions (such as sand dense forests) that might have a low local variance.

Despite of the local variance in the land regions, shores should have positive values in the binary image and sea is expected to have a low variance and therefore represented by 0 valued regions in the binary image. 1's separate the land from the sea but there may be 0's on the both sides. Selecting the largest set of connected 0's is considered for marking sea region but in some cases the 0 region in the land is larger than the 0 region on the sea and it may lead to defective detection. For this reason a human is expected to mark a point in the sea region. All 0's connected to selected point are considered as sea and all other points are marked as land

After land and sea regions are correctly marked. A simple boundary detection operation is able to determine the border as shoreline on an image that has correctly marked land and sea regions. Most of the edge detection algorithms also provide the same result with boundary tracing on a binary image.

\section{Fractal GeOMETRY IN PERFormance EVAluation}

Like most of the image recognition problems, detection of 
the shorelines are evaluated by expert opinion. The results from the algorithm are compared with the result gathered from experts. This gives a sense of how good the algorithm is performing but lacks the stability of a metric measurement.

Fractal dimension is a alternative measure for dimension that indicates how measure of objects change as the scale of measurement changes. Fractal dimension is a widely used measurement method on different kinds of data. First Mandelbrot [12] mentioned the difficulty of measuring coastlines and their fractal properties. Yu, Zhang, Wang and Yang proposed a fractal based classification for infrared iris image databases. [13] Fractal geometry is also used in a variety of geographical subjects. It is used to model the model the variant distribution of ores. It's stated that it performed better than linear geometric statistical models when a decent geological model isn't present, asymmetry is high and data is collected with irregular drilling holes.[14] Fractal properties of amorphous areas and their relation to mass movements are also studied and a computer program, FRACEK was developed for the purpose of calculating fractal dimensions. [15]

Since we know the fractal properties of shorelines, a successfully detected shoreline must preserve the fractal properties of that shore. By comparing the fractal dimension of the shores found by the expert and the algorithm we have a metric insight on how well the detection is performed.

Here fractal dimension of the shoreline is proposed as a metric on how successful the detection operation is. To perform this evaluation we need both computer generated shore and a shore that is considered to be correct. Since there is no commonly accepted algorithmic method to do so, we expect the shore that is considered as a correct representation to be generated by a human expert. Both of the generated shores is expected to have same line length to expect a correct result.

For fractals with unknown mathematical models box counting is a widely used method to determine the fractal dimension. This method is based on covering the shape with boxes of same sizes. If any part of the shape is contained by a box that box that box is taken to cover the shape. When the size of the boxes $\epsilon$ decreases, the number of boxes required to cover the shape $N(\epsilon)$ increases. Fractal dimension can be stated as: [16]

$$
d \sim \frac{\log N(\epsilon)}{\log \epsilon}
$$

Since we can find different sizes and counts for different box sizes usually $N(\epsilon)$ and $\epsilon$ is found for many box sizes. Logarithms of this values are placed on a graph with log in horizontal and $\log N(\epsilon)$ in vertical axis. Each point corresponds to values taken in a different box size. These points are then fit to a line and slope of this line is the box counting dimension for the corresponding shape.

\section{EXPERIMENT}

Proposed method is experimented on two different datasets. First one of these datasets is Satellite images of different regions of Turkey retrieved from Google Maps [17]. The dataset focuses on different geographical regions that have different geographical formations. While selecting the images quality and solidity of the images are taken into consideration. In Google Maps some regions tend to have lower image resolution than the others and image for some regions may be constructed by combining different satellite images taken in different times which can lead to certain color differentiations due to light differences. This color difference may produce a high variance where should not have occurred and may have a negative effect in our algorithm. Both of these cases are avoided during image selection.

Second dataset is the sections of The Quickbird satellite imagery product RGB composite (123) produced by Gokceoglu et al. [18]. These images are from Lara region by Mediteriansea.

Fig. 2 shows examples of images taken from Google Maps and the computer generated shoreline. The shoreline seems to overlap with the original shore in general. Because of the size of the frame used for calculating the local variances, variance map method may end up rounding up small details of bays and gulfs.

The method is applied on both Google Maps and Quickbird satellite image data sets. For each image the shoreline is also constructed separately by human expert Fractal dimension is calculated for these images.

By decreasing the frame size for variance mapping these details may be enhanced but decreasing window size has negative effect on robustness. As the resolution of the input image increases, the detail of the processed shoreline increases as well.
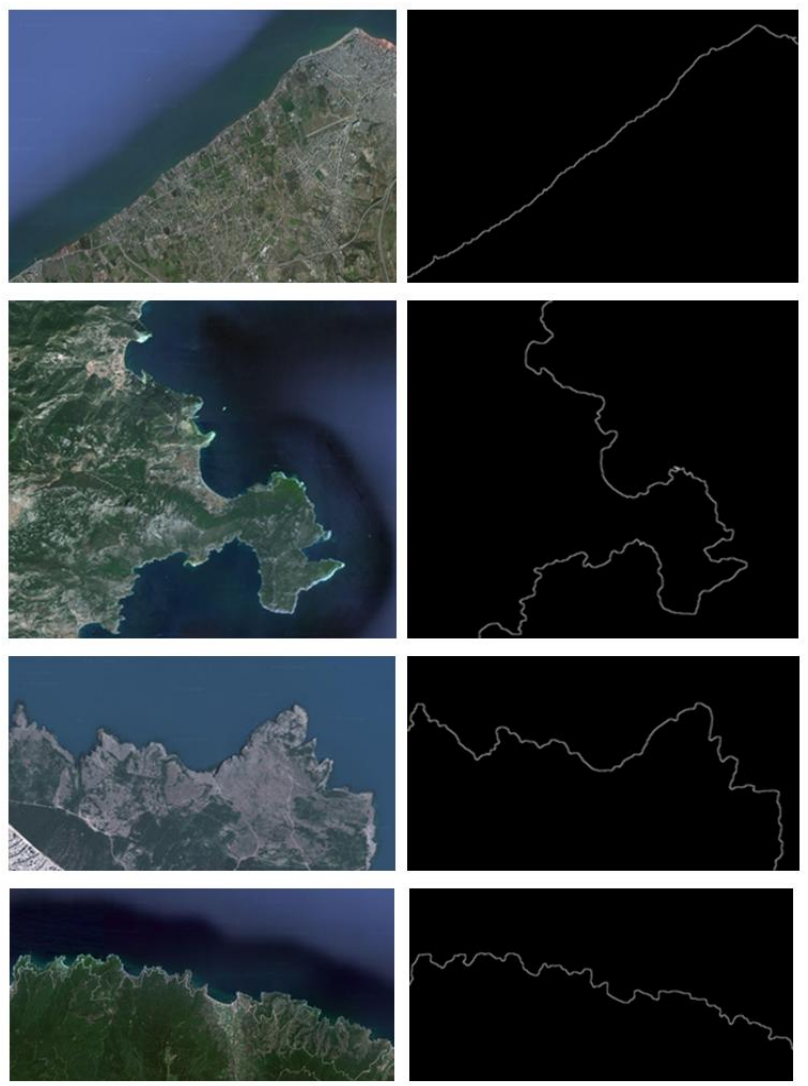

Fig. 2. Original images and extracted shorelines.

Table I and Table II give the fractal dimensions for both computer generated and human generated shorelines. The fractal dimensions are calculated by box counting method 
differ approximately between 1 and 1.2. Fractal dimensions of the computer generated shores are in general close to the fractal dimensions of human generated shores. But there are some cases that the fractal properties of the shore could not be represented by the computer generated shores.

Possible reasons for these cases are:

1) Large amount of human made objects

2) Insufficient resolution that may lead to too much rounding up.

TABLE I: FRACTAL DIMENSIONS OF COMPUTER GENERATED AND HUMAN GENERATED SHORELINES BY USING SATELLITE IMAGES

\begin{tabular}{|c|c|c|}
\hline Region & Hand Drawn & Processed \\
\hline Lara 1 & 1.0600 & 1.1165 \\
\hline Lara 2 & 0.9898 & 1.0339 \\
\hline Lara 3 & 1.0476 & 1.0589 \\
\hline Lara 4 & 1.0378 & 1.0473 \\
\hline Lara 5 & 1.0474 & 1.0471 \\
\hline
\end{tabular}

TABLE II: FRACTAL DiMENSIONS OF COMPUTER GENERATED AND HUMAN GENERATED USING GOOGLE MAPS IMAGES

\begin{tabular}{|c|c|c|}
\hline \multicolumn{1}{|c|}{ GENERATED USING GOOGLE MAPS IMAGES } \\
\hline Black Sea 1 & Hand Drawn & Processed \\
\hline Black Sea 2 & 1.0390 & 1.0517 \\
\hline Black Sea 3 & 1.1181 & 1.1091 \\
\hline Marmara 1 & 1.1292 & 1.1134 \\
\hline Marmara 2 & 1.0758 & 1.0932 \\
\hline Marmara 3 & 1.1536 & 1.0824 \\
\hline Mediterranean 1 & 1.0821 & 1.1949 \\
\hline Mediterranean 2 & 1.1434 & 1.1086 \\
\hline Mediterranean 3 & 1.0866 & 1.0802 \\
\hline Aegean 1 & 1.1728 & 1.1718 \\
\hline Aegean 2 & 1.1832 & 1.1702 \\
\hline Aegean 3 & 1.2486 & 1.1921 \\
\hline
\end{tabular}

\section{CONCLUSION}

Usually shoreline extraction performance is evaluated visually. However, representation of performance with a measurable metric rather than visual evaluation performance of extracted shoreline is of capital importance to produce comparable and interpretable results. Considering the ability of fractal geometry to express natural entities, fractal dimension is proposed to be used as a performance metric. Our contribution is measuring the performance of a automated shoreline extraction method by using fractals. Comparison is made between the calculated fractal values of extracted shoreline and man drawn shoreline. Fractal dimension is calculated by box counting method which reflects the fullest extent of possible alterations on the image on which calculations are performed, on its value. Consequently the differentiation of length of the shorelines can be reflected directly. Namely, the closer fractal dimensions of hand-drawn, accepted as gospel shoreline and automatic calculated shoreline considered as the better performance. In this study, on shore images taken from Google Maps, there are occasions where fractal dimensions are close and distant. Hereby the factors that affect the results are noise, low image resolution and amount of man-made objects. As seen in the sample pictures in Fig. 2, there is intensity and color gradient from close sea shore to offshore. These arise from changes in illumination and reflection differences of sea regions due to depth difference, shallow regions are darker then brighter ones. A common way to deal with this problem is image-smoothing which is applied in our case too. Illumination discontinuity is another determiner on result. Illumination discontinuity hides the edge discontinuities and results with wrong shoreline detection. Despite of these factors, in the study on satellite images very close fractal dimensions are observed and it is evaluated as high performance. Proposed method has a mid -level complexity.

\section{REFERENCES}

[1] S. Sural, G. Qian, and S. Pramanik, "Segmentation and histogram generation using the HSV color space for image retrieval," in Proc. International Conference on Image Processing, 2002, vol. 2, pp II-589-II-592.

[2] U. R. Aktas, G. Can, and F. T. Y. Vural, "Edge-aware segmentation in satellite imagery: A case study of shoreline detection," in Proc. Pattern Recognition in Remote Sensing 2012, Nov. 2012, pp. 1-4.

[3] Z. Y. Zhang, W. F. Feng, and J. H. Zhang, "A Coastline-detection Method Based on the Color Difference for the RS Image," Journal of Institute of Surveying and Mapping, vol. 4, pp. 7, 2005.

[4] K. Di, J. Wang, R. Ma and R. Li, "Automatic shoreline extraction from high-resolution Ikonos satellite imagery,"in Proc. ASPRS 2003 Annual Conference, vol. 3, May 2003.

[5] X. Zhang and Z. Wang, "Coastline extraction from remote sensing image based on improved minimum filter," in Proc. Second IITA International Conference on Geoscience and Remote Sensing, Aug. 2010, vol. 2, pp. 44-47.

[6] X. You and W. Li, "A sea-land segmentation scheme based on statistical model of sea", in Proc. 4th International Congress on Image and Signal Processing, Oct. 2011, vol. 3, pp. 1155-1159.

[7] N. Otsu, "A threshold selection method from gray-level histograms," Automatica, vol. 11, no. 285-296, pp. 23-27, 1975.

[8] H. Liu and K. C. Jezek, "Automated extraction of coastline from satellite imagery by integrating Canny edge detection and locally adaptive thresholding methods", International Journal of Remote Sensing, vol. 25, no. 5, pp. 937-958, 2004.

[9] J. Canny, "A computational approach to edge detection," IEEE Transactions on Pattern Analysis and Machine Intelligence, vol. 6, pp. 679-698, 1986.

[10] J. Ponce, D. Forsyth, E. P. Willow, S. Antipolis-Méditerranée, R. d'activité-RAweb, L. Inria, and I. Alumni, Computer vision: a modern approach, 2011

[11] L. Shapiro, Computer Vision and Image Processing, Academic Press., 1992.

[12] B. Mandelbrot, "How long is the coast of Britain? Statistical self-similarity and fractional dimension," Science, New Series, vol. 156, no. 3775, pp. 636-638, May 1967.

[13] L. Yu, D. Zhang, K. Wang, and W. Yang, "Coarse iris classification using box-counting to estimate fractal dimensions", Pattern Recognition, vol. 38, issue 11, pp. 1791-1798, Nov. 2005.

[14] Q. Wang, J. Deng, H. Liu, L. Yang, L. Wan, and R. Zhang, "Fractal models for ore reserve estimation," Ore Geology Reviews, vol. 37, issue 1, pp. 2-14, Feb. 2010.

[15] E. Sezer, "A computer program for fractal dimension (FRACEK) with application on type of mass movement characterization," Computers and Geosciences, vol. 36, issue 3, pp. 391-396, Mar. 2010.

[16] R. F. Voss, "Fractals in nature: from characterization to simulation," The Science of Fractal Images, Springer-Verlag, New York, pp. 21-70, 1988. 
[17] Turkey region satalite images. (April 10, 2013). [Online]. Available: http://maps.google.com

[18] C. Gokceoglu, H. A. Nefeslioglu, A. Akgun, D. Turer, and M. Temimhan, "Determination of the shore edge line at Kundu (Antalya, Turkey)," in Proc. Tenth International Conference on the Mediterranean Coastal Environment, MEDCOAST 11, E. Ozhan (Editor), Rhodes, Greece, 2011, pp. 221-228.

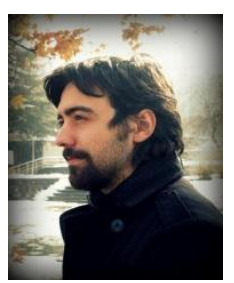

Sinan O. Altınuç was born in Niğde, Turkey in 1986. Altınuc received his B.Sc. degree from Department of Computer Engineering, Hacettepe University, Ankara, Turkey in 2010. He is a M.Sc. student at Department of Computer Engineering, Hacettepe University. His major field of study is in fractal geometry, artificial intelligence and chaotic systems.

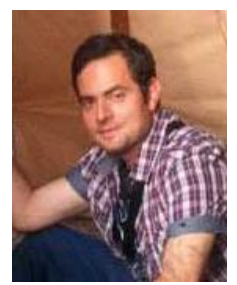

Ali S. Keçeli was born in Gaziantep, Turkey in 1984 Keçeli received his B.Sc. degree from Department of Computer Engineering, Hacettepe University, Ankara, Turkey in 2006. He received his M.Sc. degree from Department of Computer Engineering, Hacettepe University, Ankara, Turkey in 2009. He continues his $\mathrm{Ph} . \mathrm{D}$. education at the same department since 2009 . He works as a research and teaching assistant at Hacettepe University Computer Engineering Department since 2007. The author's major field of study is computer vision, data mining, image processing.

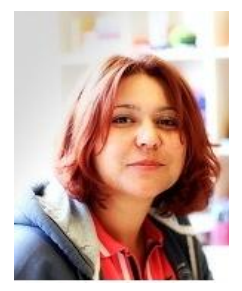

Ebru A. Sezer was born in Ankara, Turkey in 1974 received her B.Sc. degree from Department of Computer Engineering, Hacettepe University, Ankara, Turkey in 1996. She received her M.Sc. degree from Department of Computer Engineering, Hacettepe University, Ankara, Turkey in 2000. She received her Ph.D. degree from Department of Computer Engineering, Hacettepe University, Ankara, Turkey in 2006. She continues her research activities at the same department and and works as an assoc. prof. at Hacettepe University Computer Engineering Department. Her major field of study is in information retrieval, data mining, fuzzy logic. 